\title{
Performance of the ATLAS Liquid Argon calorimeter
}

\author{
Samir Arfaoui \\ samir.arfaoui@cern.ch \\ CERN/CPPM
}

on behalf of the ATLAS Liquid Argon Calorimeter Group

2010 IEEE Nuclear Science Symposium and Medical Imaging Conference

Knoxville, Tennessee, USA 


\section{Outline}

- The ATLAS Liquid Argon calorimeter

- Description

- Signal reconstruction

- Stability of calibration

- Timing alignment

- Calorimeter trigger performance

- Physics results with 7TeV collision data

- Summary 


\section{The ATLAS detector}

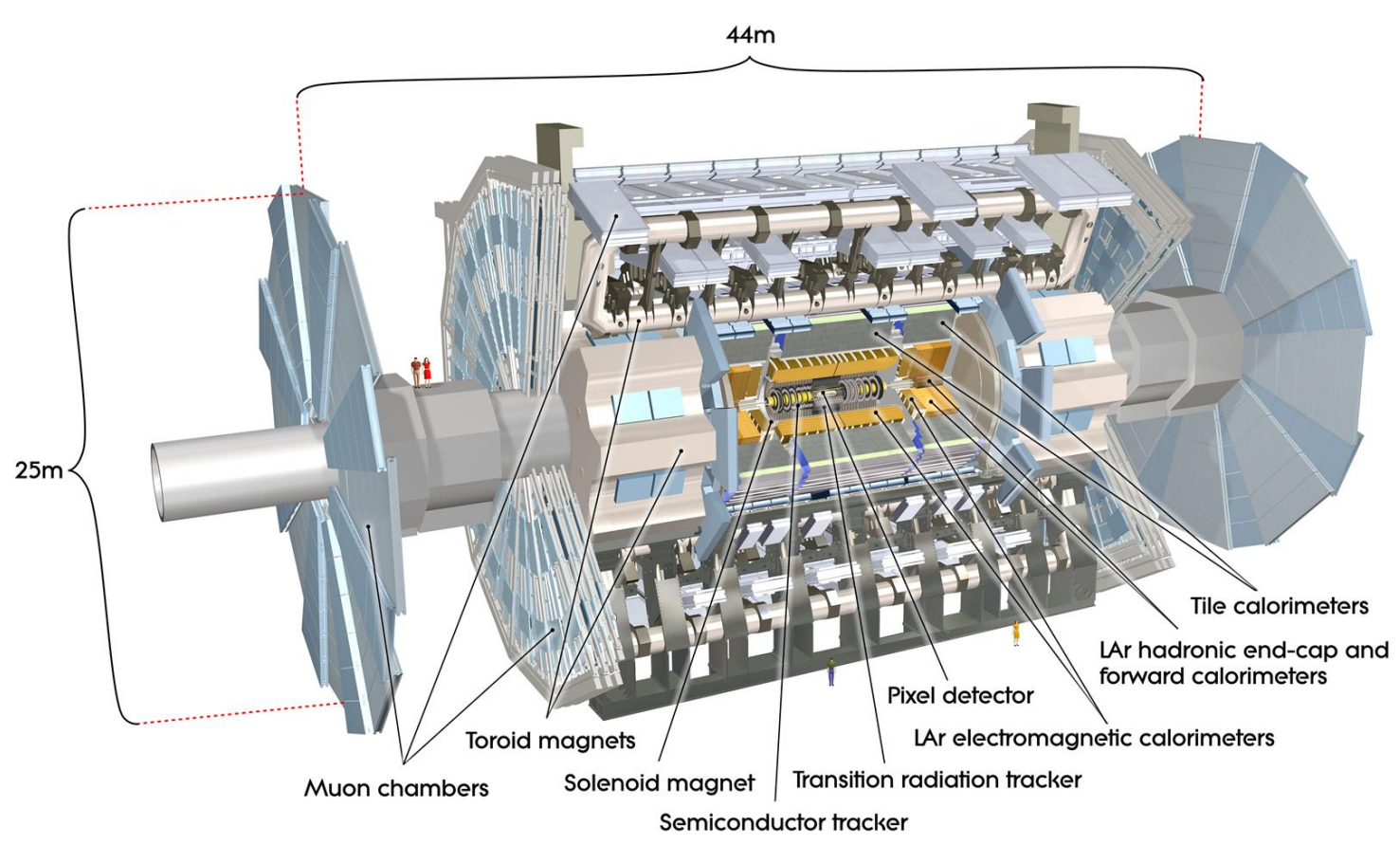

$\underline{1997}$

Start of construction, test beams 2003-2009

Installation in the experimental cavern, cosmic data

September 2008

First LHC beam

November 2009

First collisions @ 900GeV

March 2010

First collisions @7 TeV

\section{$\underline{\text { Structure }}$}

-Tracking : Pixel, Silicon detector, Transition Radiation

Tracker ; in a solenoidal magnetic field

-Calorimetry : Liquid Argon + Scintillators

- Muon spectrometer : Drift Chambers, Resistive Plate Chambers ; in a toroidal magnetic field

\section{Physics}

Standard Model, Higgs, SUSY, ...

Signatures : leptons, jets, missing transverse energy, ...

$\rightarrow$ Need for a good calorimetry! 


\section{The ATLAS LAr Calorimeter (1/2)}

Electromagnetic Calorimeter (EM)
- Absorbers : Pb
- Active Medium : LAr
- Accordion geometry : full $\phi$ coverage
- Coverage $:|\eta|<3.2$
- Segmentation in $\eta$ and in depth
- 3 layers up to $|\eta|=2.5 ; 2$ up to $|\eta|=3.2$
$\quad$ - Layer $1: \Delta \eta \times \Delta \phi=0.0031 \times 0.1$
$\quad$ - Layer $2: \Delta \eta \times \Delta \phi=0.025 \times 0.025$
- Presampler up to $|\eta|=1.8$
- 173312 readout channels $(98.5 \%$ operational)
- Design resolution : $\frac{\Delta E}{E}=\frac{10 \%}{\sqrt{E(G e V)}} \oplus 0.7 \%$
(from test beam)
- Photon angular resolution : $\approx \frac{50 \mu r a d}{\sqrt{E(G e V)}}$

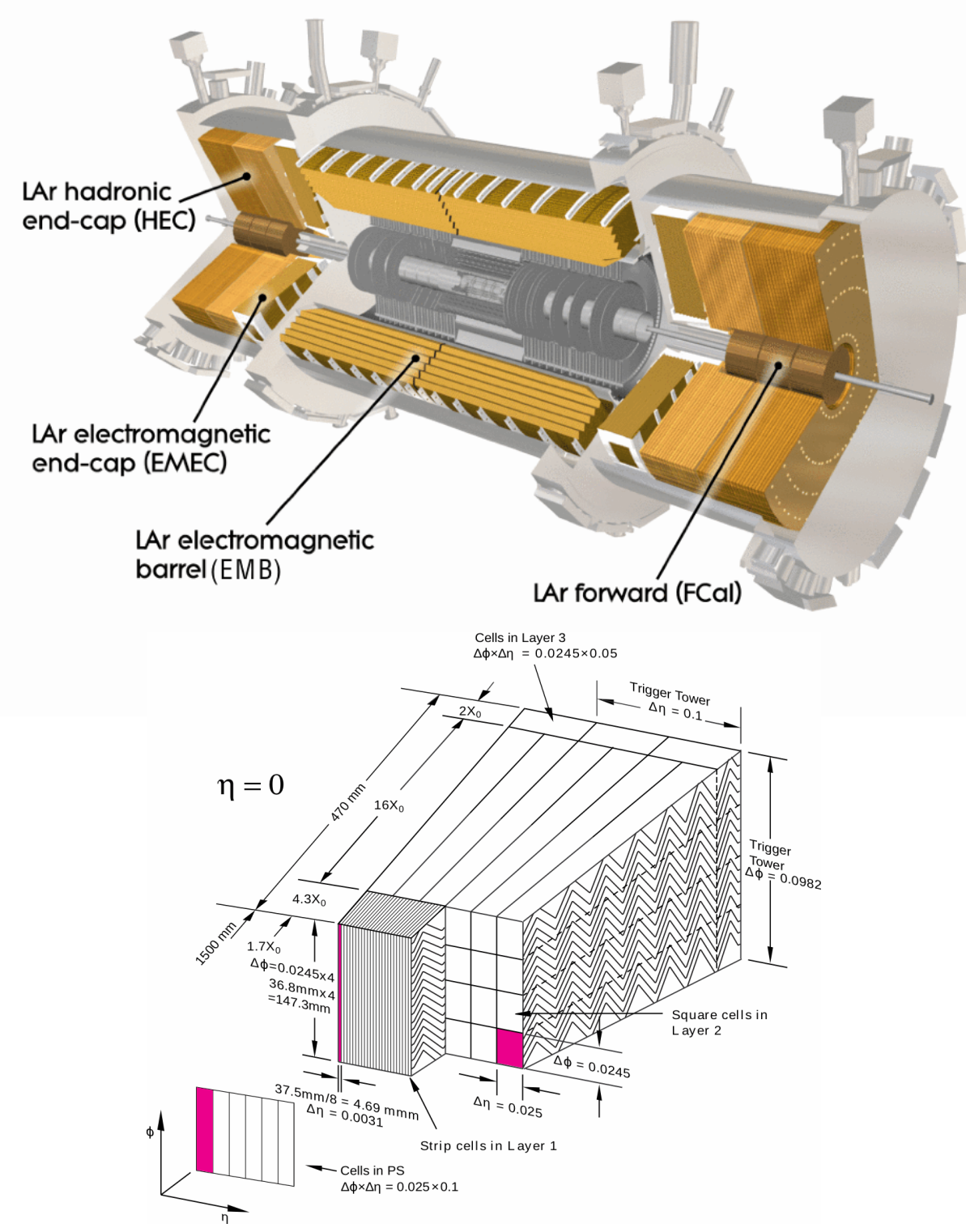




\section{The ATLAS LAr Calorimeter (2/2)}

$$
\begin{aligned}
& \text { Hadronic Endcap (HEC) } \\
& \text { - Absorbers : } \mathrm{Cu} \\
& \text { - Active medium : LAr } \\
& \text { - Coverage }: 1.5<|\eta|<3.2 \\
& \text { - } 4 \text { layers }(\Delta \eta \times \Delta \phi=0.1 \times 0.1 \text { or } 0.2 \times 0.2 \text { ) } \\
& \text { - } 5632 \text { readout channels }(99.9 \% \text { operational) } \\
& \text { - Design resolution : } \frac{\Delta E}{E}=\frac{50 \%}{\sqrt{E(G e V)}} \oplus 3 \%
\end{aligned}
$$

Forward Calorimeter (FCal)
- Absorbers : Cu/W
- Active medium : LAr
- Coverage : $3.1<|\eta|<4.9$
- $1 \mathrm{EM}+2$ Hadronic layers
- 3524 readout channels ( $100 \%$ operational)
- Design resolution : $\frac{\Delta E}{E}=\frac{100 \%}{\sqrt{E(G e V)}} \oplus 10 \%$
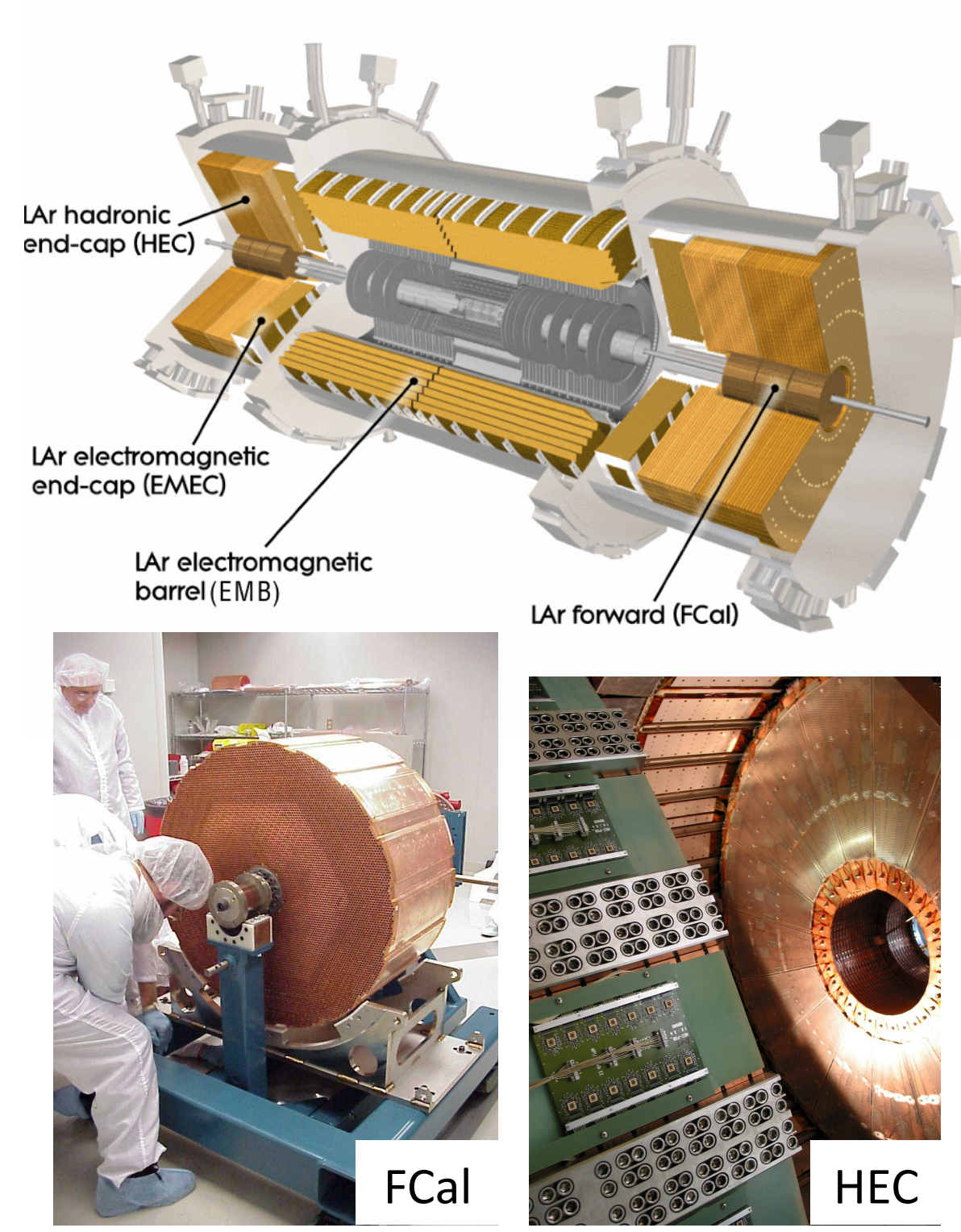


\section{Signal reconstruction}

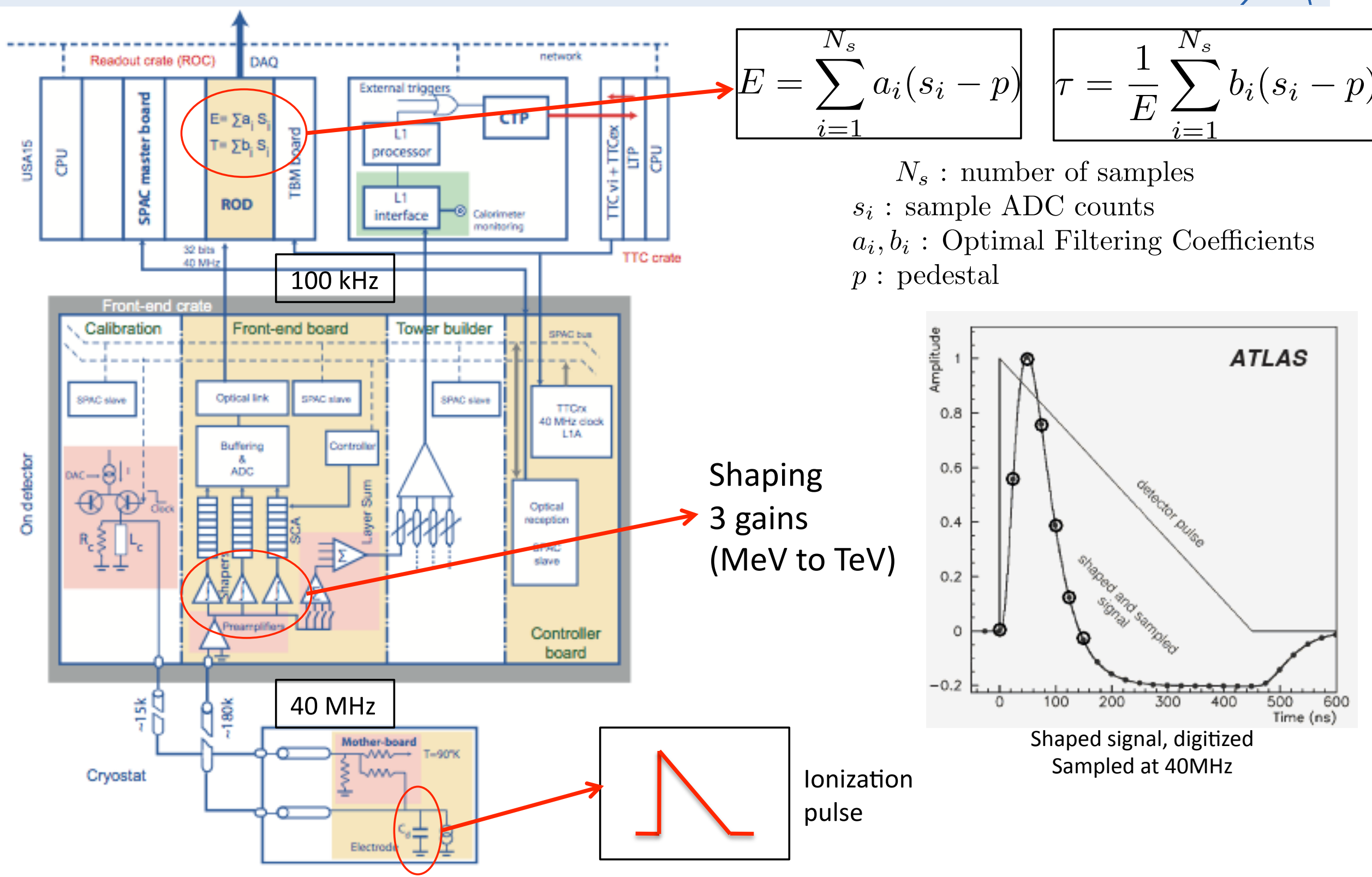




\section{Stability of calibration}
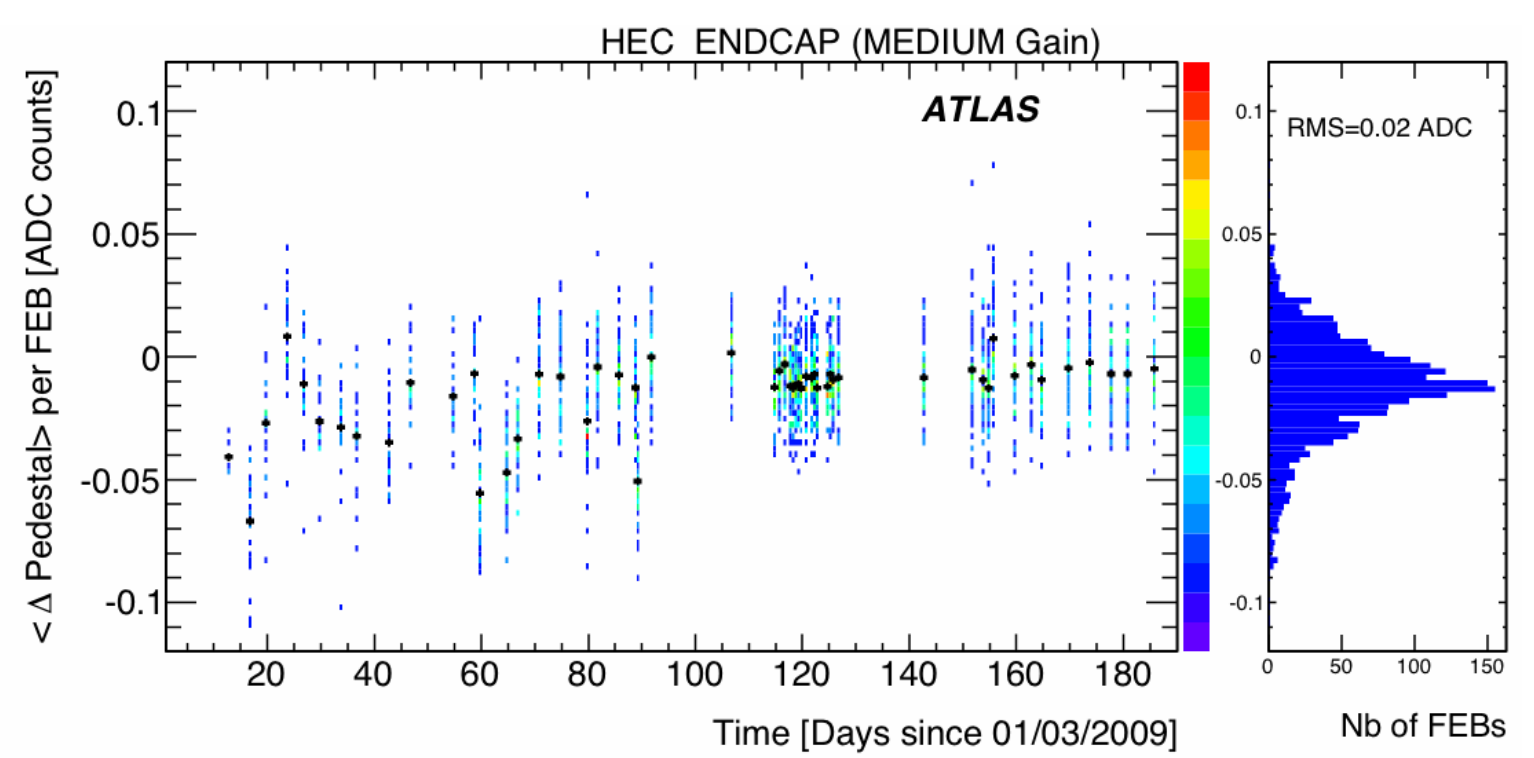

\section{Stability of calibration constants}

- Calibration runs are taken between every LHC fill

- Calibration constants are updated every few weeks

- Stability of constants are

monitored over long periods (here 6 months in 2009)

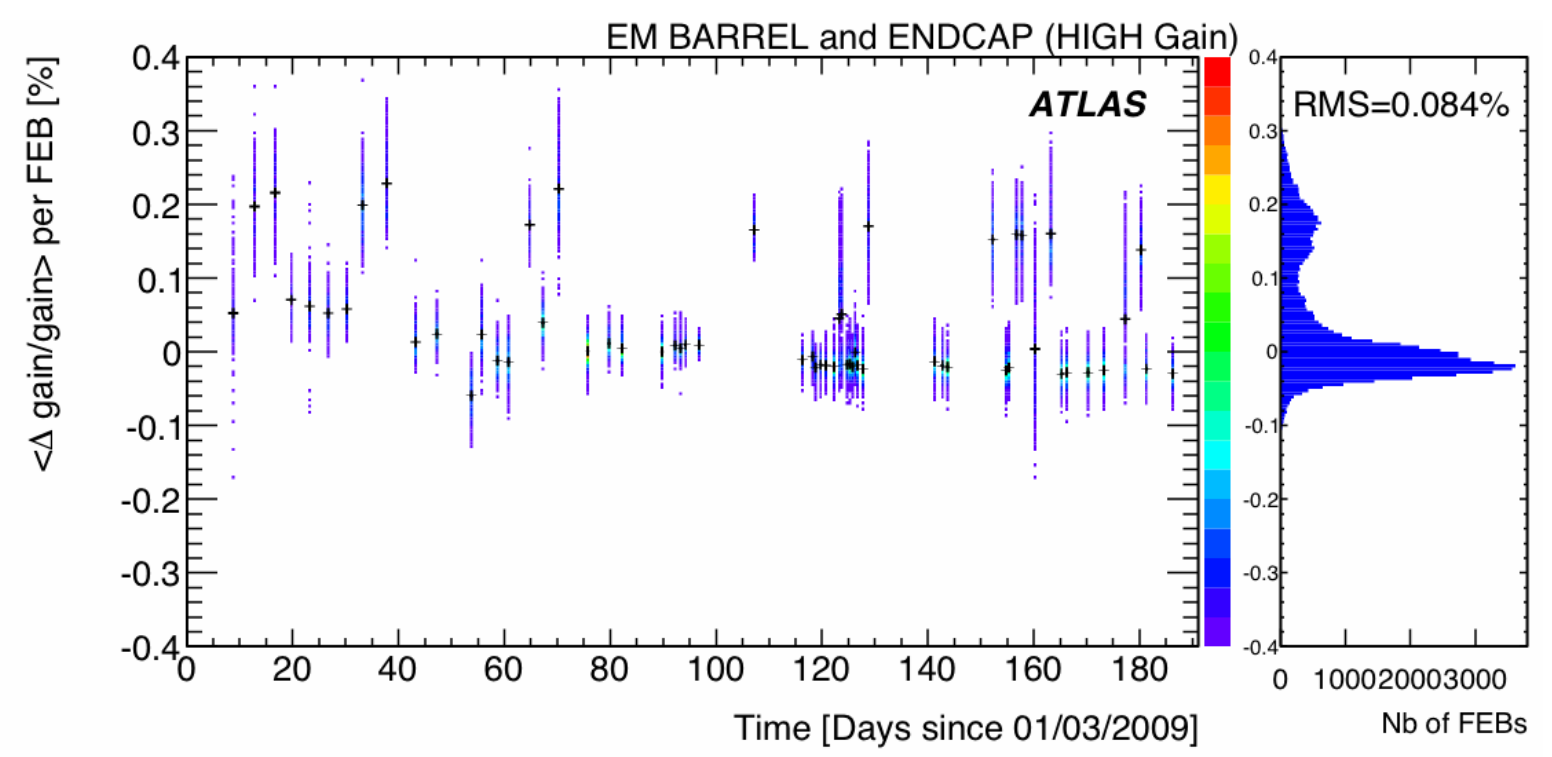

- Pedestals : $<0.03$ ADC counts for all calorimeters

- Gains : $<0.1 \%$ for all calorimeters

$\rightarrow$ Robust calibration procedure

$\rightarrow$ Good electronics stability 


\section{Timing alignment}
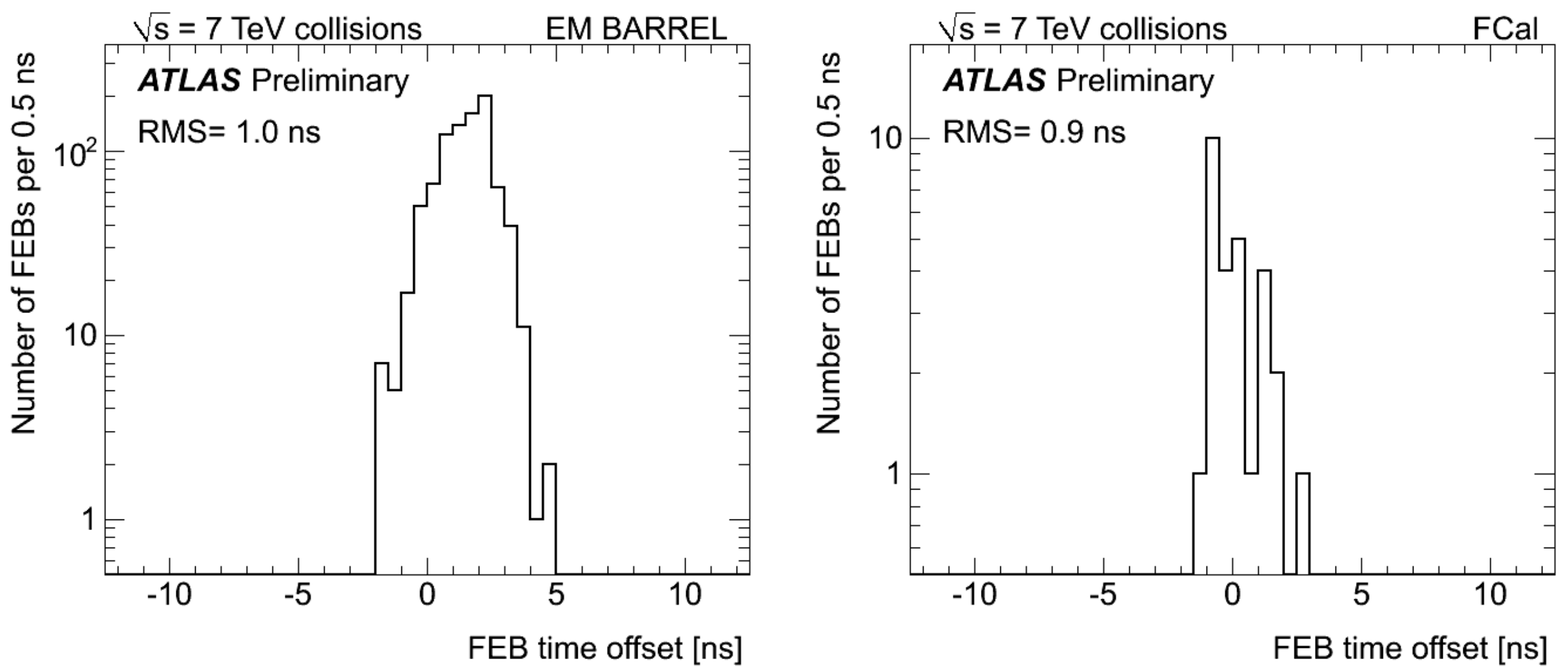

Goal : align all the readout electronics in time Prediction : time of flight, cabling length, ... Time is calculated with collision data along with energy Above : offset between calculated time and prediction

$\rightarrow$ Achieved so far : 1ns per frontend board (128 readout channels) $\rightarrow$ Goal : 100ps per readout channel 


\section{Trigger performance}

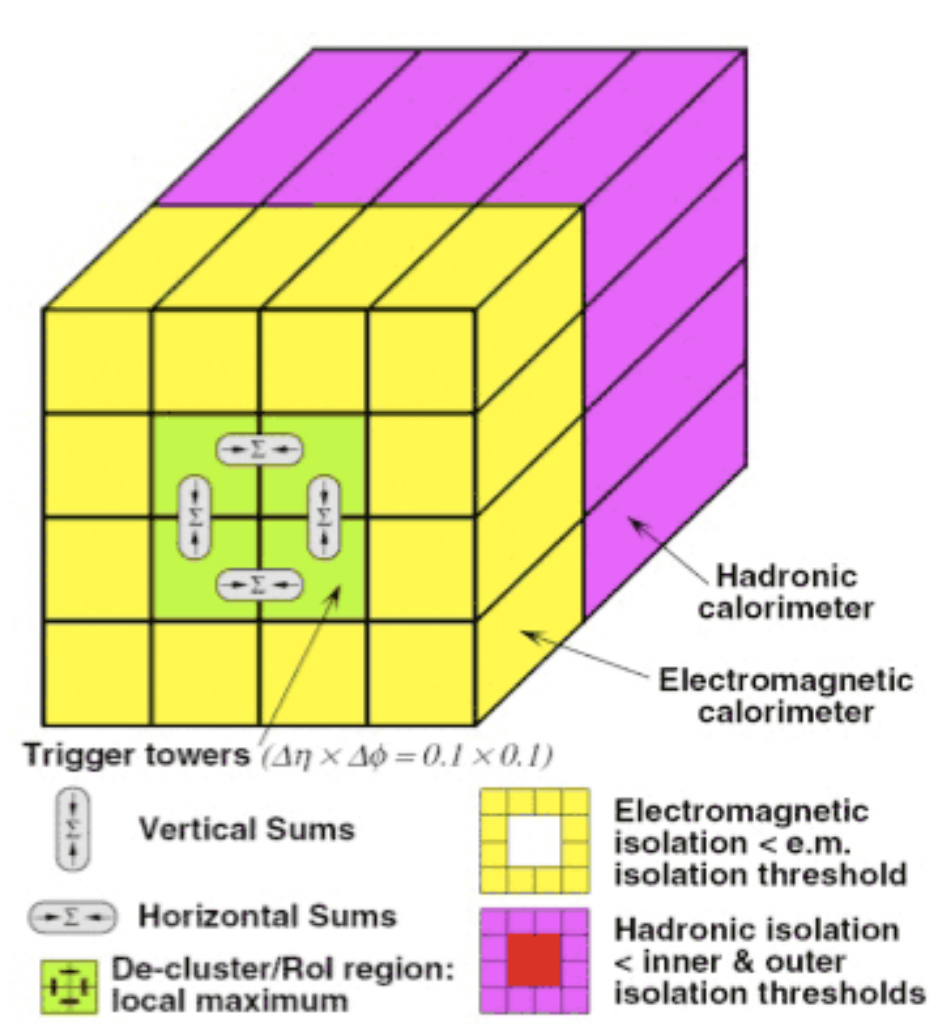

- Energy is summed over neighboring cells in trigger tower

- Typical size : $\Delta \eta \times \Delta \phi=0.1 \times 0.1$ (approx. 60 readout channels)

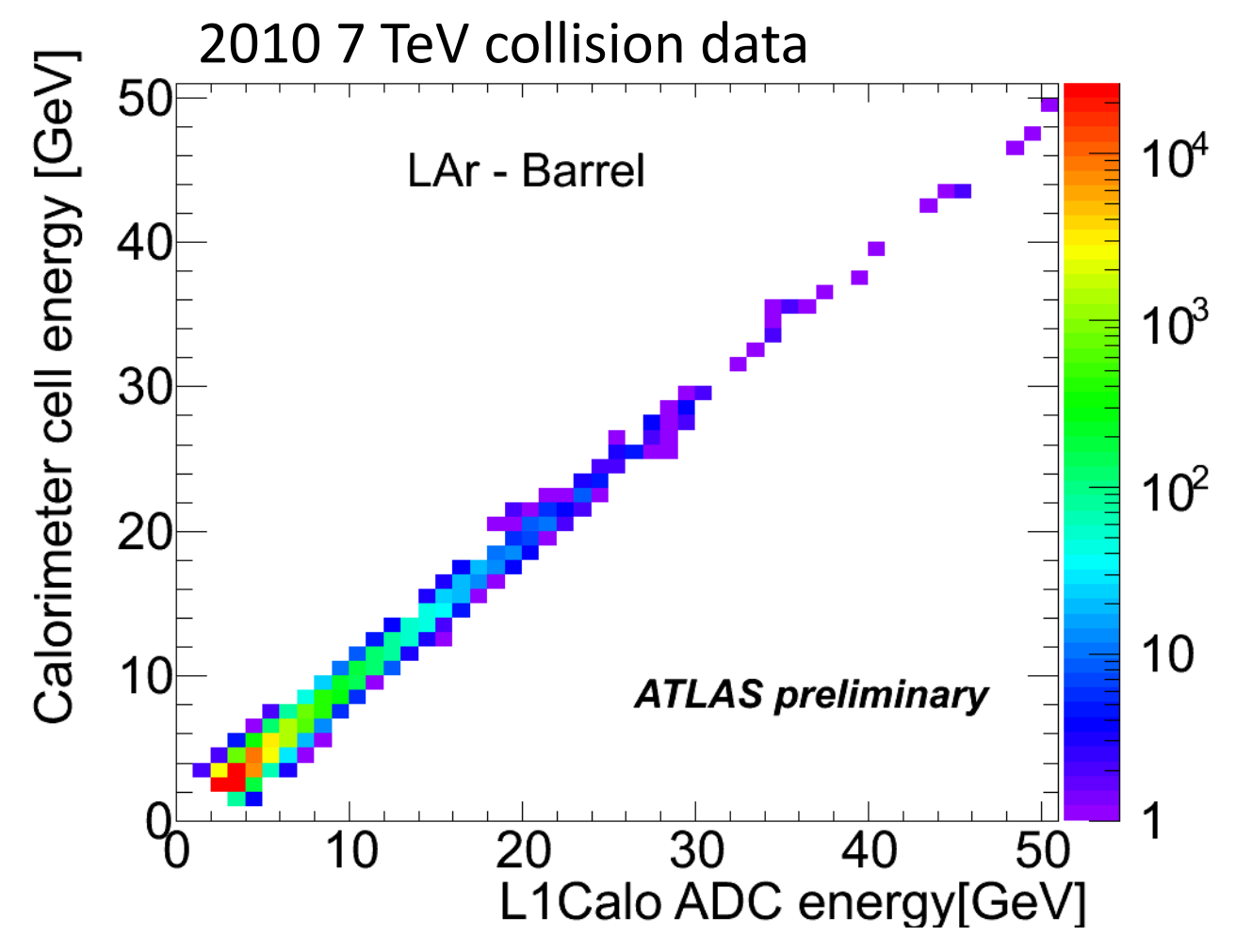

- Trigger energy can be used to correct energy when digital readout is missing

Resolution of $\mathrm{E}_{\mathrm{T}}(\mathrm{L}$ 1Calo $)<5 \%$ for $\mathrm{E}_{\mathrm{T}}(\mathrm{LAr})>10 \mathrm{GeV}$ 


\section{Physics results (1/2)}

Photon identification results
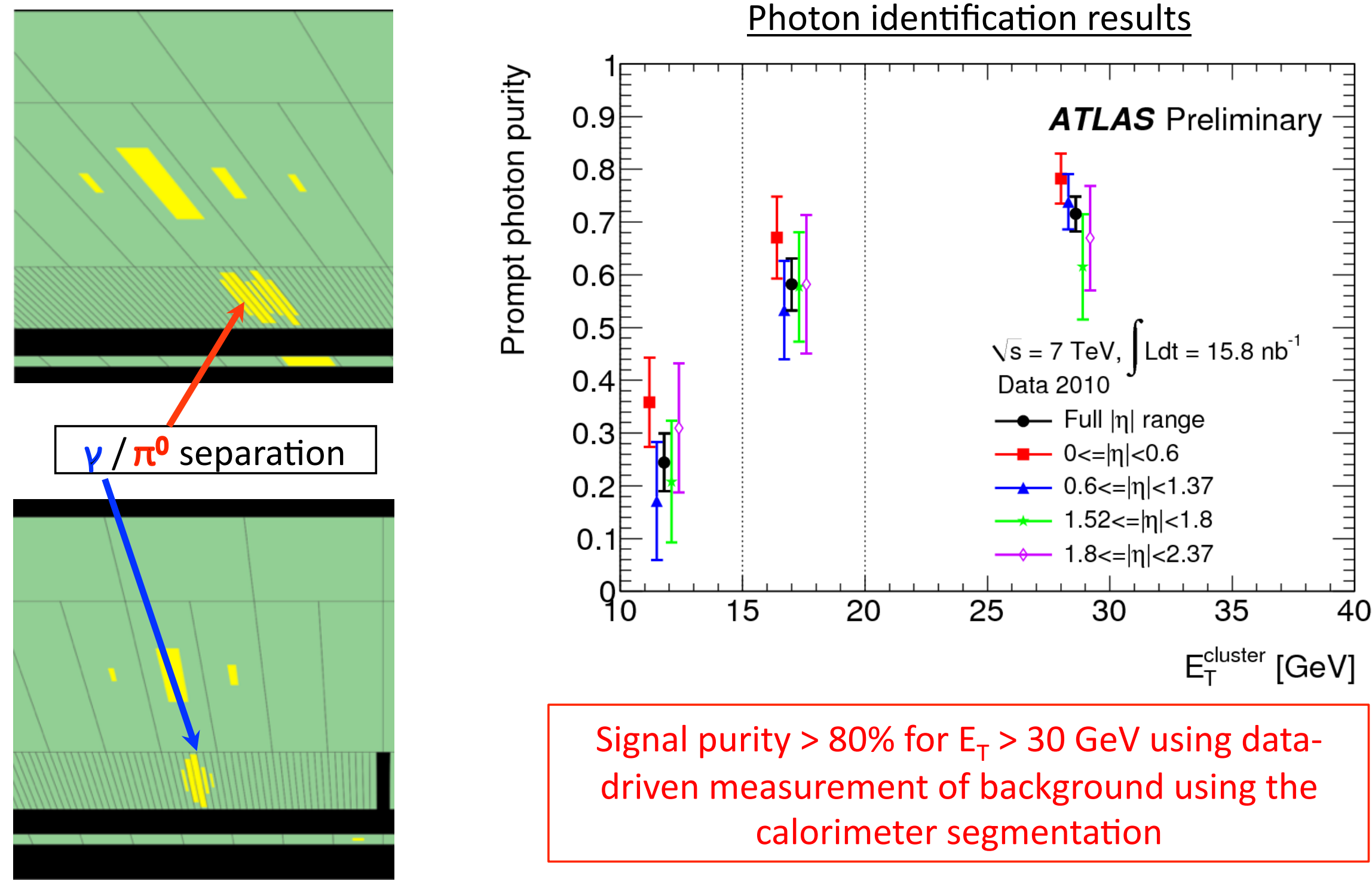

Signal purity $>80 \%$ for $\mathrm{E}_{\mathrm{T}}>30 \mathrm{GeV}$ using datadriven measurement of background using the calorimeter segmentation 


\section{Physics results (2/2)}
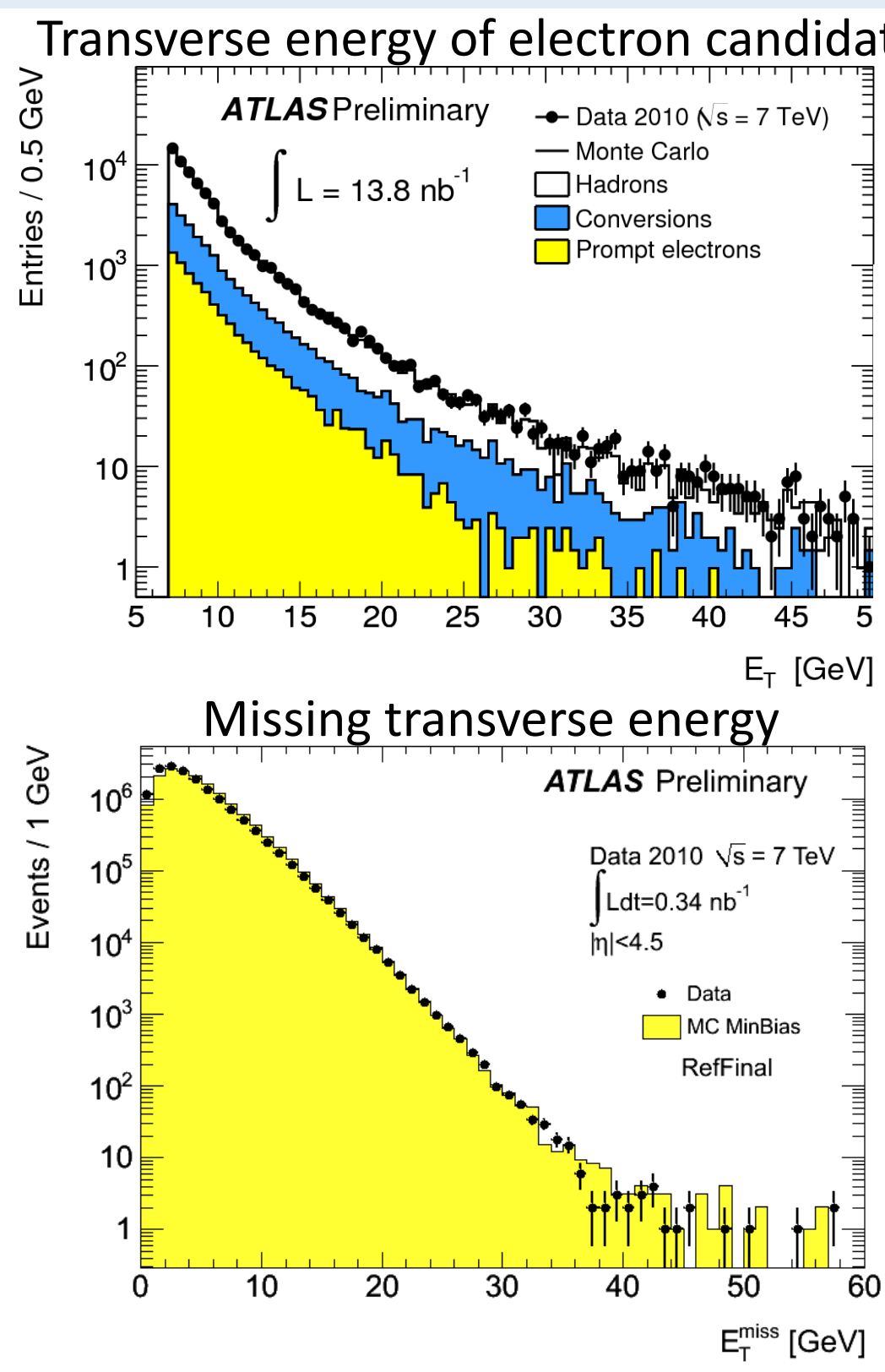

$\rightarrow$ Good agreement between data and Monte-Carlo for variables related to physics objects
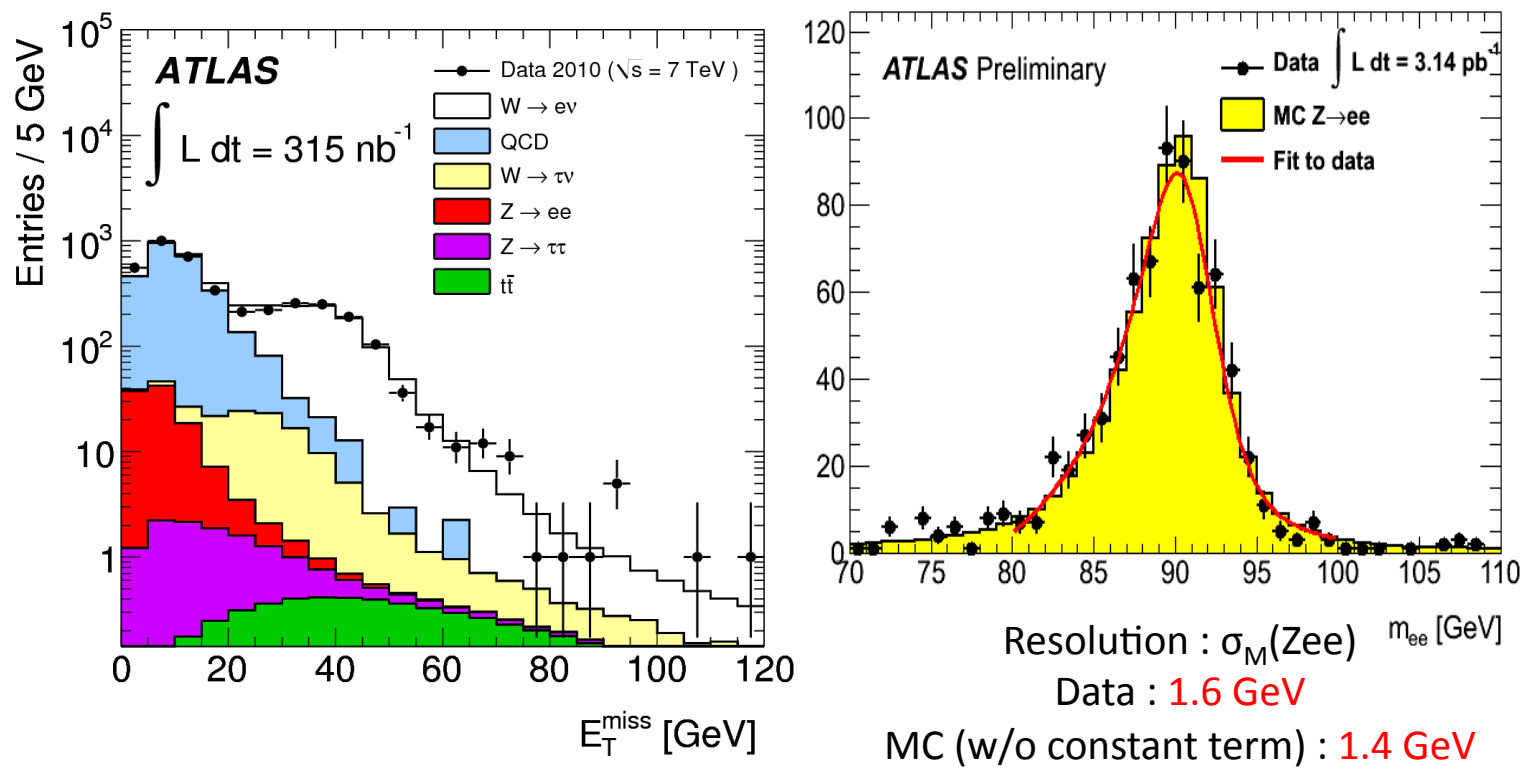

Above : missing transverse energy for $\mathrm{W}$->ev candidates (left) and invariant mass of Z->ee candidates (right)

$\rightarrow$ Well understood calorimeter variables allow interesting rediscoveries! 


\section{Summary}

- The ATLAS Liquid Argon Calorimeter is installed in the cavern since 2006

- Intense commissioning with cosmic muons until first LHC collisions in 2009

- Calibration

- Procedure is robust, constants are stable over long periods

- Updates every few weeks if necessary

- Timing alignment

- Achieved : a few 100 ps

- Goal : 100 ps

- Basic calorimeter variables are well understood and under control

- Electrons, photons, missing transverse energy

- First physics results very promising

- $W, Z$, jets cross-section measurements

\section{LAr Calorimeter is well unsterstood Performance is very close to design}




\section{Thank you}




\section{ATLAS design resolutions}

\begin{tabular}{|l|l|c|c|}
\hline Detector component & \multicolumn{1}{|c|}{ Required resolution } & \multicolumn{2}{c|}{$\eta$ coverage } \\
& & Measurement & Trigger \\
\hline Tracking & $\sigma_{p_{T}} / p_{T}=0.05 \% p_{T} \oplus 1 \%$ & \pm 2.5 & \\
\hline EM calorimetry & $\sigma_{E} / E=10 \% / \sqrt{E} \oplus 0.7 \%$ & \pm 3.2 & \pm 2.5 \\
\hline $\begin{array}{l}\text { Hadronic calorimetry (jets) } \\
\text { barrel and end-cap } \\
\text { forward }\end{array}$ & $\begin{array}{l}\sigma_{E} / E=50 \% / \sqrt{E} \oplus 3 \% \\
\sigma_{E} / E=100 \% / \sqrt{E} \oplus 10 \%\end{array}$ & $3.1<|\eta|<4.9$ & $3.1<|\eta|<4.9$ \\
\hline Muon spectrometer & $\sigma_{p_{T}} / p_{T}=10 \%$ at $p_{T}=1 \mathrm{TeV}$ & \pm 2.7 & \pm 2.4 \\
\hline
\end{tabular}

Table 1. General performance goals of the ATLAS detector. Note that, for high- $p_{T}$ muons, the muonspectrometer performance is independent of the inner-detector system. The units for $E$ and $p_{T}$ are in $\mathrm{GeV}$. 


\section{LAr in numbers}

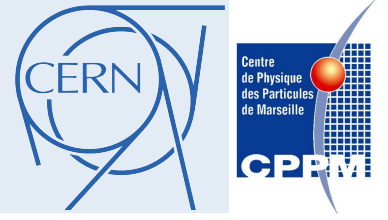

\section{Granularity}

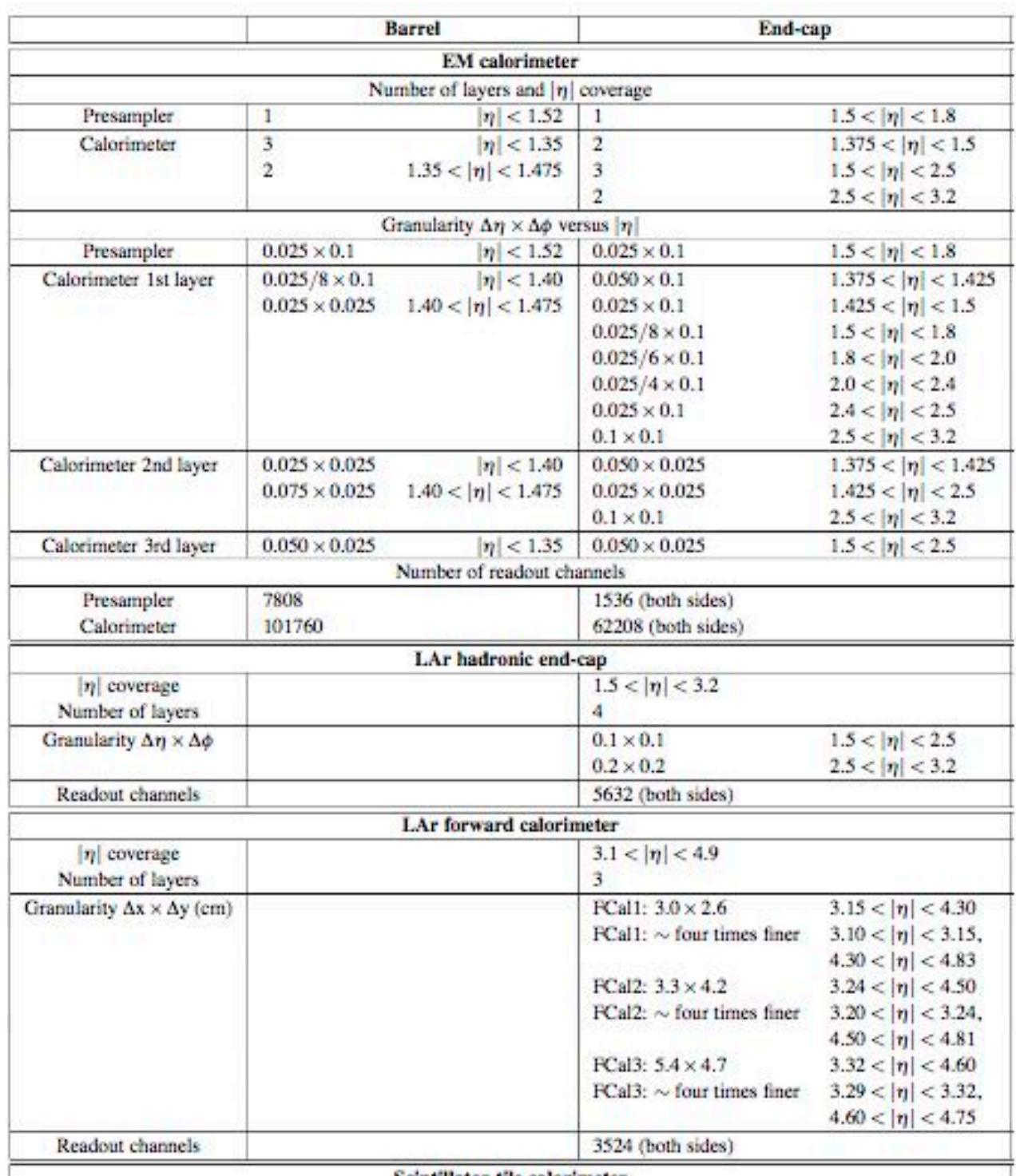

\section{Desgin channel count}

\begin{tabular}{|l|c|c|}
\hline Layer & $\begin{array}{c}\text { Number } \\
\text { of } \\
\text { channels }\end{array}$ \\
\cline { 1 - 1 } EM Barrel \\
\cline { 1 - 1 } Presampler & 7808 \\
Layer 1 & 57216 & \\
Layer 2 & 28672 & \\
Layer 3 & 13824 & \multirow{2}{*}{ Total EM Barrel } \\
Barrel end & 2048 & 109568 \\
Calibration & 8192 & \\
\cline { 1 - 1 } EM Endcap & \\
\cline { 1 - 1 } Presampler & 1536 & \\
Layer 1 & 28544 & \\
Layer 2 & 23424 & \multirow{2}{*}{ Total EM Endcap } \\
Layer 3 & 10240 & 63744 \\
Calibration & 5952 &
\end{tabular}

\begin{tabular}{|c|c|c|c|}
\hline $\begin{array}{l}\text { HEC module and } \\
\text { section }\end{array}$ & $\begin{array}{c}\text { Total number } \\
\text { of channels }\end{array}$ & Module & Total number \\
\hline HEC1 (first section) & 1536 & & of channels \\
\hline HECl (second section) & 1472 & $\mid$ FCall (side A) & 1008 \\
\hline HEC2 (first section) & 1344 & $\mid$ FCal2 (side A) & 500 \\
\hline HEC2 (second section) & 1280 & $\mid$ FCal3 (side A) & 254 \\
\hline Calibration & 1024 & & \\
\hline \multicolumn{2}{|c|}{$\begin{array}{c}\text { Total HEC } \\
5632\end{array}$} & $\left|\begin{array}{l}\text { FCal2 (side C) } \\
\text { FCal3 (side C) }\end{array}\right|$ & $\begin{array}{l}500 \\
254\end{array}$ \\
\hline & & \multicolumn{2}{|c|}{$\begin{array}{c}\text { Total FCal } \\
3524\end{array}$} \\
\hline & $\begin{array}{r}\text { rand Tot } \\
18246\end{array}$ & & \\
\hline
\end{tabular}




\section{Energy reconstruction}

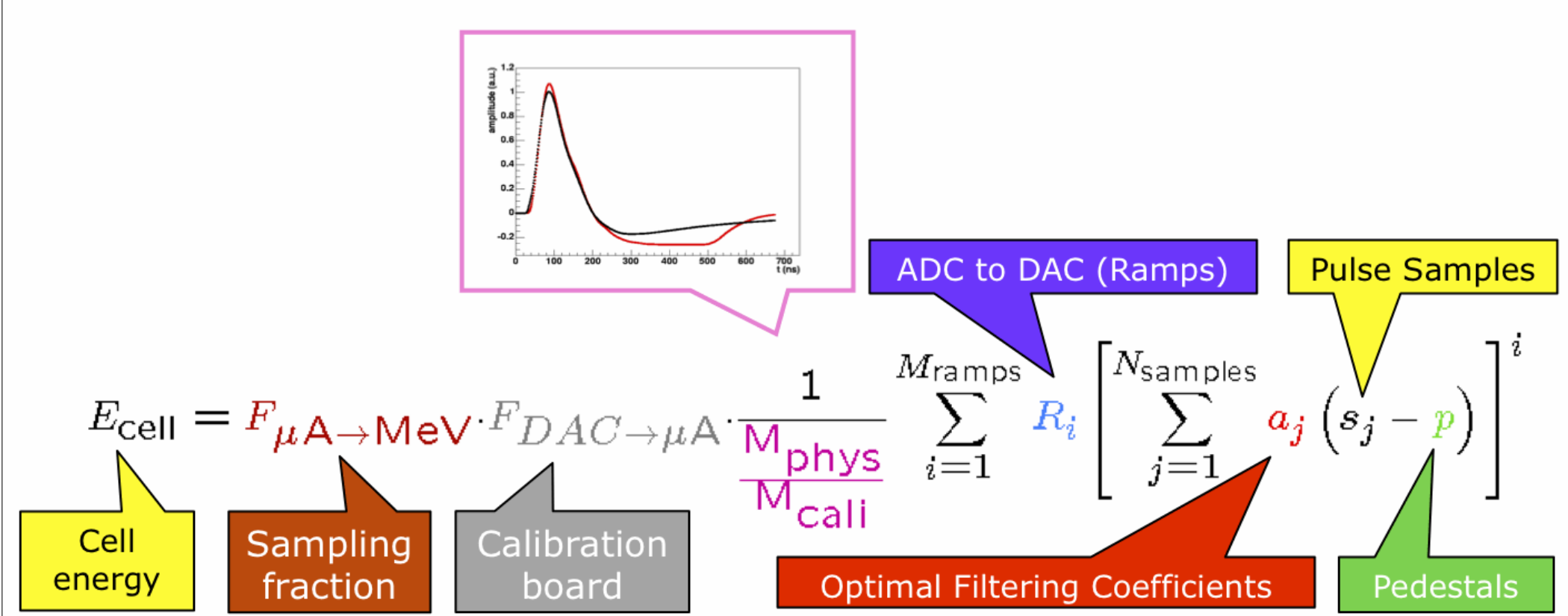

The above formula describe the LAr electronic calibration chain (from the signal ADC samples to the raw energy in the cell. Note that this version of the formula uses the general $\mathrm{M}_{\text {ramps }}$-order polynomial fit of the ramps. Actually we just use a linear fit (electronic is very linear, and additionally we only want to apply a linear gain in the DSP in order to be able to undo it offline, and apply a more refined calibration). In this case, the formula is simply:

$$
E_{\text {Cell }}=F_{\mu \mathrm{A} \rightarrow \mathrm{MeV}} \cdot F_{D A C \rightarrow \mu \mathrm{A}} \cdot \frac{1}{\frac{\mathrm{M}_{\text {phys }}}{\mathrm{M}_{\text {Cali }}}} \cdot R\left[\sum_{j=1}^{N_{\text {samples }}} a_{j}\left(s_{j}-p\right)\right]
$$

